\title{
Commentary on
}

Values-Focused Exposure and Response Prevention in the Treatment of Comorbid Schizophrenia and Obsessive-Compulsive Disorder: The Case of "Mr. H, and

"Functional," Sub-Clinical Obsessive-Compulsive Symptoms and Their Challenges: The Case of "Angela"

\section{Considerations for Modifying Exposure and Response Prevention: The Cases of "Mr. H" and "Angela"}

\author{
DAVID AUSTERN ${ }^{\mathrm{a}, \mathrm{c}}$, TANYA FARBER ${ }^{\mathrm{a}, \mathrm{b}, \mathrm{c}}$, \& JAMES MARINCHAK $^{\mathrm{a}}$ \\ a Anxiety Disorders Clinic, Rutgers-The State University of New Jersey \\ ${ }^{\mathrm{b}}$ Correspondence regarding this article should be addressed to Tanya Farber, Anxiety Disorders Clinic, 797 Hoes \\ Lane West, Piscataway, NJ 08854-8020 \\ ${ }^{\mathrm{c}}$ Authors Austern and Farber contributed equally to this article. \\ Email: tfarber@eden.rutgers.edu
}

\begin{abstract}
Exposure and response prevention (ERP) is considered the gold standard treatment for obsessivecompulsive disorder (OCD). However, when comorbidity or other clinically relevant issues arise, the standard ERP protocol may require modification. Golden and Holaway's (2013) case of "Mr. $\mathrm{H}$ " and Pontes and Pereira's (2013) case of "Angela" offer excellent examples of clients presenting with these clinical challenges. Our commentary addresses some of the concerns raised by these authors by bringing in the emerging field of Distress Tolerance (DT) research. Using examples from the authors' cases, along with relevant research, we suggest that clinicians who wish to modify ERP consider the proposed alterations carefully by simultaneously thinking more broadly and more specifically about the psychopathology present. In addition, we stress the necessity of various forms of continual outcome monitoring.
\end{abstract}

Key words: exposure therapy; exposure and response prevention (ERP); obsessive-compulsive disorder (OCD); anxiety; distress tolerance (DT); comorbidity; case studies; clinical case studies

The case studies of "Mr. H" presented by Golden and Holaway (2013) and of "Angela" presented by Pontes and Pereira (2013) demonstrate how the treatment of obsessive-compulsive disorder (OCD) symptoms can be complicated by the presence of comorbid disorders and other clinically relevant issues. The potential for such complications is considerable; according to the National Comorbidity Survey Replication (NCS-R), approximately 90\% of the individuals who had met criteria for an OCD diagnosis at one point in their lives also suffered from another disorder (Ruscio, Stein, Chiu, \& Kessler, 2010). Over 50\% of respondents who had lifetime OCD also suffered from one or more comorbid anxiety disorders, mood disorders, or impulse- 
control disorders. In a separate study, approximately one third of subjects who suffered from OCD also had an Axis II diagnosis (Steketee, Chambless, \& Tran, 2001).

In addition to diagnosable disorders, personal and environmental characteristics such as religiosity, perfectionism, and familial accommodation influence the individual's subjective experience of OCD as well as the course and effectiveness of treatment. Research suggests that clients receiving treatment for OCD who also had major depressive disorder (MDD) or personality traits that fall into the anxious, dramatic, or odd categories experienced fewer benefits from exposure and response prevention (ERP) (Steketee et al., 2001). In particular, more symptoms of paranoid, schizoid, and schizotypal personality disorders were associated with worse treatment outcome, although this relationship may have been due to an overall greater level of psychopathology (de Haan et al., 1997).

The case studies included in this issue illustrate the specific ways in which comorbid disorders and real-world issues outside of the therapy office can affect OCD and its treatment. The authors go further to present the rationale for certain modifications to ERP, as well as describe the implementation and outcomes of the adapted treatment. While such alterations may prove helpful for individuals with particular needs associated with other problems, the changes must be clearly justified and designed based on the ERP model and evidence-based practice as well as clinical considerations. When modifying an empirically-supported treatment, the clinician must delineate a clear "guiding conception" that directs the adapted interventions (Persons, 2008).

\section{ADAPTING ERP FOR OCD WITH ASSOCIATED CLINICAL ISSUES: CHALLENGES}

In the cases of Mr. $\mathrm{H}$ and Angela, the clinicians encountered unique challenges to the treatment of OCD symptoms and related clinical issues. Afflicted with schizophrenia, $\mathrm{Mr}$. $\mathrm{H}$ demonstrated psychotic symptoms in session, including paranoid delusions as well as severe agitation. This not only restricted the amount of insight he was capable of achieving relative to the true effects of his OCD, but also limited the time he was able to engage in ERP to approximately 20 minutes. His disorganized presentation may have also prevented him from independently initiating exposures for homework.

Angela did not meet criteria for OCD, although she did report struggling with some obsessive thoughts. Perhaps due to her depressive symptoms and the limited nature of her OCD symptoms and associated distress and impairment, she evidenced low motivation for treatment.

Other challenges that may arise in treatment with clients who have comorbid disorders or other clinically relevant issues include difficulty understanding the rationale for ERP, treatment refusal due to a belief that the client cannot withstand exposure, and the covert use of neutralizing behaviors during exposure (Salkovskis et al., 2003). However, with these challenges come opportunities to implement ERP in creative and effective ways with clients who may nonetheless benefit from the treatment. 


\section{ADAPTING ERP FOR OCD WITH ASSOCIATED CLINICAL ISSUES: OPPORTUNITIES}

In the cases of Mr. $\mathrm{H}$ and Angela, the therapists seized upon several opportunities provided by the specific circumstances of the clients. Although $\mathrm{Mr}$. $\mathrm{H}$ was not able to autonomously initiate homework exposures, his board-and-care operator collaborated with the therapist to plan exposures between sessions. The availability of an interested and reliable third party to initiate exposures and help the client resist the urge to ritualize likely enhanced client compliance with homework, a critical element of ERP. In addition, the persistent delusions held by Mr. H provided the basis for a goal that motivated him to pursue ERP and experience gains. Ethical questions aside, the presence of a problem area like Mr. H's delusion may be assessed and potentially utilized to create a motivating goal for the client.

When working with OCD clients with comorbid depression, ERP can also be implemented in a way that increases the individual's contact with reinforcing experiences, such as Angela's increased interaction with her husband and family. An examination of secondary gain obtained from OCD symptoms can also be used to create alternative opportunities for the client to gain similar reinforcement in more adaptive ways. By making explicit the behavioral contingencies maintaining the OCD symptoms, the therapist can also help the client assume a more active role in recognizing, understanding, and modifying these contingencies. Such were the alterations to ERP made by Golden and Holaway and by Pontes and Pereira. Basing their interventions on the principles of ERP and the clinical condition of their clients, the therapists confronted the challenges associated with each case and created opportunities for expanding treatment. However, such modifications carry with them certain risks.

\section{ADAPTING ERP FOR OCD WITH ASSOCIATED CLINICAL ISSUES: RISKS AND POTENTIAL BENEFITS}

The most significant risk stems from the relative lack of evidence supporting the efficacy of ERP when used to treat OCD with comorbid schizophrenia, depression, or other clinically relevant issues (Wilson, 1997). Although the mechanisms of habituation and new learning about one's ability to manage distress may still apply, the conditions associated with these disorders may affect response to treatment. Specific to the case of $\mathrm{Mr}$. H, another risk associated with the use of a delusion as a motivating goal for the client may be the possibility that colluding with a delusion in this manner may serve to inadvertently reinforce the false belief, which may affect future treatment of the schizophrenia.

In the case of Angela, the therapist's decisions to avoid placing a tentative end date on treatment, to discontinue addressing the issue of homework noncompliance, and to focus on the client's relationship with her husband rather than her obsessions may have prolonged the ERP treatment. In altering an evidence-based treatment, a therapist sometimes chooses to pursue alternative gains, such as increased behavioral activation, which may come at the expense of rapid improvement in OCD symptoms. However, the benefits of adapting treatment to the individual needs of the client, when combined with measures that can be taken to ensure the 
most ethical and effective implementation of the modified interventions, may make the approach clinically justifiable.

Such potential benefits include the client's enhanced sense of self-efficacy, increased distress tolerance, and skillful use of adaptive coping strategies, as well as the expected reduction of distressing obsessive thoughts and compulsions. Secondary to these gains, individuals may experience increases in independence and global functioning, as well as a reduction in the persistence and intensity of personal distress. In both the cases of $\mathrm{Mr}$. $\mathrm{H}$ and Angela, the clients had sufficient awareness of their active roles in the exposure to attribute a successful instance of managing anxiety to personal capability and skill. Furthermore, the expansion of exposure to include more forms of physical contact between Angela and her family members led her to engage in reinforcing interactions with others, as well as tolerate the associated anxiety. Ultimately, the availability of an effective treatment for OCD offers both clinicians and clients an opportunity to take an empirically-supported treatment and utilize it to improve an individual's immediate quality of life and understanding of personal skills and capacity. The potential benefits may lead therapists to attempt to adapt ERP in a manner like that described in Mr. H's and Angela's cases.

Nonetheless, when such alterations to an empirically-supported treatment are made, the demonstrated efficacy of the treatment may not translate into its modified form. Therefore, extreme caution must be exercised. To promote ethical and effective practice in these instances, we recommend several measures. We would urge the therapist to monitor outcome and process consistently (utilizing intervention ratings, behavioral measures, and standardized instruments), as well as to engage in continuous critical refection to ensure that all treatment adaptations are rooted in a theoretically grounded understanding of the client's problems. One way to do this is to utilize an evolving formulation based on ERP principles as well as a thorough conceptualization of the client's characteristics, environment, and problems to help direct the course of treatment, as illustrated in Persons (2008). A problem-solving model may be helpful in systematically assessing these variables and the functional relationships between them, and using this information to guide clinical decisions (Nezu, Nezu, Friedman, \& Haynes, 1997). Within this approach, a clinician may wish to examine the impact of ERP and its altered components on the client's distress tolerance.

\section{DISTRESS TOLERANCE, INTOLERANCE OF UNCERTAINTY, AND OCD}

Though distress tolerance (DT) is likely a familiar term to most readers, recent research has started to examine the links between the DT construct and psychopathology. DT is defined as the perceived capacity to withstand negative emotional/physical states and the behavioral act of withstanding these aversive internal states; as such, it is hypothesized to contribute to both the etiology and maintenance of numerous psychological disorders (Leyro, Zvolensky, \& Bernstein, 2010). While Leyro and colleagues have attempted to synthesize the conceptually and operationally heterogeneous DT literature, several researchers (e.g. Cougle, Timpano, Fitch, \& Hawkins, 2011) have specifically focused on the role DT plays in developing and maintaining the obsessive symptoms in OCD, finding support for a critical relationship between DT and 
obsessions. The growing research on the clinical impact of DT suggests that psychological treatments for a variety of diagnoses should target DT. Therefore, it is worth examining to what extent Golden and Hollaway (2013) and Pontes and Pereira (2013) modified the standard Exposure and Response Prevention (ERP) protocol to target the DT construct, albeit unintentionally.

Leyro, Zvolensky, and Bernstein (2010) note that the DT construct may be hierarchical and composed of several lower-order factors, such as Intolerance of Uncertainty (IU), Tolerance of Ambiguity (TOA), and Ability to Withstand Negative Emotional States. Historically, constructs like IU and TOA have been used interchangeably by researchers, but recent studies have revealed differences between them (Grenier, Barrette, \& Ladouceur, 2005). Grenier and colleagues used the distinction that IU refers to uncertain, future-oriented events while TOA refers to uncertain events in the "here and now." For example, someone with Social Phobia may attend a party and experience anxiety if they assume other party goers are whispering about them, which would reflect low ambiguity tolerance (TOA) of the stimulus of whispering. In addition, they may also be reluctant to initiate a conversation with others due to their uncertainty of how the other party goers will respond, which would reflect a high intolerance of uncertainty (IU). Unfortunately, the disentangling of these lower-order DT constructs is just beginning.

As Leyro and colleagues (2010) indicate, IU is most commonly associated with Generalized Anxiety Disorder (GAD), but it has also been linked to the etiology and maintenance of OCD. In a variety of experiments (e.g., Tolin et al., 2003; Holaway, Heimberg, \& Coles, 2006), IU has been linked to the phenomenology of pathological doubt and checking behaviors. If someone with OCD experiences an intrusive thought about forgetting to perform an action, that person's level of IU may moderate the likelihood of performing a checking behavior to neutralize the obsession and decrease distress. Though it is somewhat unclear whether IU is implicated in all forms of OCD (e.g., washing rituals) or is uniquely associated with pathological checking, Tolin et al. (2003) recommend modifying the standard ERP protocol to incorporate IU into in-vivo and imaginal exposures by purposefully having patients confront feelings of uncertainty and doubt.

\section{DISTRESS TOLERANCE, ASSESSMENT, AND OUTCOME MONITORING}

Additionally, Tolin and colleagues (2003) advise therapists to assess whether their OCD clients' fears are related to IU, which has typically been assessed via self-report measures like the Intolerance of Uncertainty Scale (IUS-12; Carleton, Norton, \& Asmundson, 2007). Unfortunately, the cases of Mr. $\mathrm{H}$ and of Angela both presented challenges with data collection and outcome monitoring. In the case of Mr. H, outcome measures (e.g., the Y-BOCS) were not given due to the client's “severe level of agitation.” Ironically, it was Mr. H's poor distress tolerance that made it impossible to measure his distress tolerance!

Pontes and Pereira note the importance of analyzing the function of OCD compulsions, which may vary depending on the client, because an ERP manual treats all compulsions equally by instructing clients to prevent the response from happening. We agree this is important, as it 
also may shed light on which lower-order dimension of distress tolerance the client may be deficient. For example, if Angela washes her hands after her husband returns home because she fears her husband may have touched one of his brother's objects, this suggests Angela may have both high IU and low TOA. As Angela does not know if her husband has been contaminated, making her husband an ambiguous stimulus, she may reduce this ambiguity by perceiving her husband as a contaminated, unambiguous stimulus. This reflects a preference for clarity over ambiguity in the present-moment, or low TOA. Furthermore, as she does not know the potential outcome of that hypothetical contamination, she may not be able to tolerate the distress of an uncertain future. She may reduce this uncertainty by assuming that if she is contaminated, a catastrophic outcome will definitely occur, which reflects high IU. In contrast to those hypotheses, if she washes to neutralize feelings of guilt after experiencing an intrusive thought about her brother-in-law, this suggests Angela may have a low ability to withstand a negative emotional state because she just wants the guilt to disappear. As this clinical example illustrates, it seems worthwhile for therapists to assess which lower-order DT constructs to target specifically.

Fortunately, self-reports and questionnaires are not the only techniques in our toolbox to assess and monitor outcomes. Golden and Holaway wisely relied on the board-and-care home staff to provide ongoing behavioral data as the therapy progressed. This allowed them access to objective indicators of Mr. H's behavioral change, which Mr. $\mathrm{H}$ himself largely failed to provide due to his comorbid schizophrenia diagnosis. At post-treatment, the board-and-care operator was able to confirm a significant reduction in time spent washing and a significant reduction in the frequency of requests to wash. This is excellent quantitative data, and also there is qualitative data that suggests increased distress tolerance. For example, Mr. H’s audiologist reported a marked decrease in "freaking out" when the audiologist touched Mr. H's hearing aids at posttreatment compared to pre-treatment. "Freaking out," in particular, seems to epitomize the behavioral response clients could engage in when they cannot withstand a negative emotional state. Additionally, Mr. H reportedly demonstrated an increase in frustration tolerance when he fastened his seatbelt by himself as opposed to first expressing frustration about other residents in the board-and-care home, which he had done previously. Note that frustration tolerance is hypothesized as another one of the lower-order constructs that comprise the DT construct (Leyro, Zvolensky, \& Bernstein, 2010).

Mr. H’s lack of neutralizing responses (i.e., his lack of compulsions and lack of "freaking out") at post-treatment leads to several possibilities about mechanisms of therapeutic change. Has he habituated to the anxiety generated within and between exposure sessions and thus extinction has occurred? Does he still experience distress (i.e., an aversive emotional/physical state) yet behaviorally persist without seeking a negative reinforcement opportunity (i.e., compulsions and “freaking out”)? Defining DT as the refraining of responding to a negative reinforcement opportunity (Trafton \& Gifford, 2011) speaks to the heart of ERP, as the goal is for our clients to "behaviorally persist” by turning down negative reinforcement opportunities. Unfortunately, Mr. H’s lack of insight into his own behavioral change makes answering this question quite difficult. 


\section{DISTRESS TOLERANCE, FOLLOW-UP DATA, AND GENERALIZABILITY}

If we had access to follow-up data for $\mathrm{Mr}$. $\mathrm{H}$ and Angela, we would be better equipped to talk about mechanisms of change in their exposures and what type of learning occurred. Perhaps $\mathrm{Mr}$. $\mathrm{H}$ has resumed his washing behaviors since post-treatment data was collected. If so, this could raise questions about the efficacy of doing ERP for OCD within the context of a delusional system. It seems intuitive that treatment gains would be tougher to maintain over time when the client may only have participated in the treatment for the purposes of secondary gain. However, if the treatment gains have been maintained then this could suggest that real learning has occurred. While we are aware that collecting follow-up data can be challenging and occasionally impossible, if authors like Golden and Holaway were able to collect relevant follow-up data in their cases, this could shed light on some of these questions.

Follow-up data may also help to shed light on whether a particular lower-order construct of DT may have been most salient in maintaining Mr. H's distress, or whether targeting any lower-order construct generalizes to the entire DT construct. A hallmark of the rationale for exposure therapy is the phenomenon whereby treatment gains made during the completion of specific exercises will diffuse to a variety of contexts and environments outside the therapy office (Abramowitz, 2006). For example, a client with a list of hundreds of distinct household checks does not need to do in vivo or imaginal exposures for each item on the checklist; the assumption is that by targeting several of them the learning will generalize across situations. It is possible that designing exposures targeting lower-order DT constructs such as IU or TOA generalizes to the entire DT construct. As seen with $\mathrm{Mr}$. $\mathrm{H}$, he appeared to have increased his frustration tolerance at post-treatment even though frustration-tolerance was never explicitly targeted during the ERP protocol. Further research with non-psychotic populations may be needed to obtain qualitative sources of post-treatment and follow-up data about what types of DT increases they may be experiencing.

\section{CONCLUSION}

These cases illustrate clinical opportunities to move beyond treatment manuals by adjusting for comorbidities and other clinically relevant issues. When treating OCD using ERP in the context of other clinical issues, this might require the clinician to consider how the principles and practice of ERP may also help enhance a client's DT via exposures. When presented with one of these challenging cases, we advise clinicians to simultaneously think more broadly and more specifically about psychopathology. The higher-order construct of DT is one way of broadly conceptualizing a pathological process common to multiple psychological disorders.

In the case of Mr. H, the clinicians found a way to modify ERP for a client with comorbid schizophrenia by thinking broadly about distress. As Mr. H reported greater distress from his OCD than from his delusional system, conducting ERP had great clinical utility. And yet, clinicians must also think more specifically about psychopathology when presented with a challenging case, like that of Angela. Angela's compulsions served multiple functions, which 
required the therapist to adopt a more nuanced approach than an ERP manual may teach. By isolating which lower-order DT construct is implicated in each compulsion, clinicians can design more specific and targeted exposures. Armed with the ability to conceptualize the case both broadly and specifically, clinicians can create more comprehensive treatments that are rooted in empirical evidence and theory as well as adapted to the needs of the individual client.

\section{REFERENCES}

Abramowitz, J. S. (2006). In Review. Canadian Journal of Psychiatry, 51, 407-416.

Carleton, R. N., Norton, M. A., \& Asmundson, G. J. (2007). Fearing the unknown: A short version of the Intolerance of Uncertainty Scale. Journal of Anxiety Disorders, 21(1), 105- 117.

Cougle, J. R., Timpano, K. R., Fitch, K. E., \& Hawkins, K. A. (2011). Distress tolerance and obsessions: an integrative analysis. Depression and anxiety, 28(10), 906-914.

de Haan, E., van Oppen, P., van Balkom, A. J. L. M., Spinhoven, P., Hooduin, K. A. L., \& van Dyck, R. (1997). Prediction of outcome and early vs. late improvement in OCD patients treated with cognitive behaviour therapy and pharmacotherapy. Acta Psychiatrica Scandinavica, 96, 354-361.

Golden, A., \& Holaway, R. M. (2013). Values-focused exposure and response prevention in the treatment of comorbid schizophrenia and obsessive-compulsive disorder: The case of "Mr. H." Pragmatic Case Studies in Psychotherapy, 9(1), Article 3, 65-80. Available: http://hdl.rutgers.edu/1782.1/pcsp_journal

Grenier, S., Barrette, A. M., \& Ladouceur, R. (2005). Intolerance of uncertainty and intolerance of ambiguity: Similarities and differences. Personality and individual differences, 39(3), 593-600.

Holaway, R. M., Heimberg, R. G., \& Coles, M. E. (2006). A comparison of intolerance of uncertainty in analogue obsessive-compulsive disorder and generalized anxiety disorder. Journal of Anxiety Disorders, 20(2), 158-174.

Leyro, T. M., Zvolensky, M. J., \& Bernstein, A. (2010). Distress tolerance and psychopathological symptoms and disorders: a review of the empirical literature among adults. Psychological bulletin, 136(4), 576.

Nezu, A. M., Nezu, C. M., Friedman, S. H., Haynes, S. N. (1997). Case formulation in behavior therapy: Problem-solving and functional analytic strategies. In T. D. Eells (Ed.), Handbook of psychotherapy case formulation (pp. 368-401). New York, NY: Guilford Press.

Persons, J. B. (2008). The case formulation approach to cognitive-behavior therapy. New York: Guilford Press.

Pontes, M. M., \& Pereira, R. F. (2013). "Functional,” sub-clinical obsessive-compulsive symptoms and their challenges: The Case of "Angela." Pragmatic Case Studies in Psychotherapy, 9(1), Article 4, 81-97. Available: http://hdl.rutgers.edu/1782.1/pcsp_journal

Ruscio, A. M., Stein, D. J., Chiu, W. T., \& Kessler, R. C. (2010). The epidemiology of obsessive-compulsive disorder in National Comorbidity Survey Replication. Molecular Psychiatry, 15(1), 53-63. 
Salkovskis, P. M., Thorpe, S. J., Wahl, K., Wroe, A. L., \& Forrester, E. (2003). Neutralizing increases discomfort associated with obsessional thoughts: An experimental study with obsessional patients. Journal of Abnormal Psychology, 112(4), 709-715.

Steketee, G., Chambless, D. L., \& Tran, G. Q. (2001). Effects of Axis I and II comorbidity on behavior therapy outcome for obsessive-compulsive disorder and agoraphobia. Comprehensive Psychiatry, 42(1), 76-86.

Tolin, D. F., Abramowitz, J. S., Brigidi, B. D., \& Foa, E. B. (2003). Intolerance of uncertainty in obsessive-compulsive disorder. Journal of Anxiety Disorders, 17(2), 233-242.

Trafton, J. A., \& Gifford, E. V. (2011). Biological bases of distress tolerance. In M.J. Zvolensky, A. Bernstein, \& A.A. Vujanovic (Eds.). Distress tolerance: Theory, research, and clinical applications (pp. 80-102). New York: Guilford Press.

Wilson, G. T. (1997). Treatment manuals in clinical practice. Behaviour Research and Therapy, 35(3), 205-210. 